\title{
Resonance Characteristics and Effective Parameters of New Left Hand Metamaterial
}

\author{
Rajni $^{{ }^{* 1}}$, Anupma Marwaha ${ }^{2}$ \\ ${ }^{* 1}$ Dept. of Electronics and Communication Eng., Shaheed Bhagat Singh College State Technical Campus, \\ Ferozepur, Punjab, India. Ph./Fax: 91-9779190066/1632-242138 \\ ${ }^{2}$ Dept. of Electronics and Communication Eng., Sant Longowal Institute of Eng. and Technology, \\ Longowal, Sangrur, Punjab, India Ph./Fax: 91-9872224055/1672-253117 \\ ${ }^{*}$ Corresponding author, email: rajni_c123@yahoo.co.in ${ }^{1}$, marwaha_anupma@yahoo.co.in²
}

\begin{abstract}
It is essential to alter effective electromagnetic parameters of a material to enhance its response. In the present work, we propose a novel Left Hand Metamaterial (LHM) structure comprising double turn spiral resonator (DTSR) and capacitance loaded strips (CLS). This structure is numerically explored to examine the resonance and effective material parameters i.e. permeability and permittivity. The negative refraction in the unit cell is confirmed with identification of double negative region (negative permittivity, $\varepsilon$ and negative permeability, $\mu$ ) on placing the unit cell in a waveguide with well defined Perfect Electric Conduction/Perfect Magnetic Conduction boundary conditions.
\end{abstract}

Keywords: left hand metamaterial (LHM), spiral resonator (SR), negative permittivity, negative permeability, high frequency structure simulator (HFSS)

Copyright $(2015$ Institute of Advanced Engineering and Science. All rights reserved.

\section{Introduction}

There has been a great deal of attraction and attention in design of structures based materials in last decade, which exhibit new unnatural qualitative response functions. A recent example of these artificial materials is 'Metamaterials' which have led to paradigm shifting by opening prospects for improved antenna design to overpower the limitations of conventional antennas. These materials demonstrate negative permittivity and/or negative permeability. Metamaterials are unnatural materials that could be engineered by embedding specific inclusions of metal in some host media. This ensuant material can be tailored to achieve electromagnetic characteristics (such as permeability and permittivity) according to system requirements [1]. Due to their exotic features, these materials have applications in the field of antenna to design small antennas [2] with enhanced directivity [3] and beam-width control [4]. Another application of metamaterials is to enhance the magnetic permeability of nonmagnetic materials by metallic inclusions [5].

The man behind the remarkable discovery of these materials was Victor Veselago [6] who, in 1968, made a theoretical assumption of artificial materials which exhibit negative permittivity and permeability. His work was acknowledged after more than three decades when Pendry et al. proposed periodical thin-wire (TW) structure that exhibits the negative effective permittivity [7]. It was also demonstrated in [8] that negative magnetic permeability could be achieved using an array of split-ring resonators (SRR). In 2001, Smith realized the first prototype Left Hand Metamaterial (LHM) structure by combining split ring resonators and thin wires [9]. LHMs have numerous exceptional properties particularly the backward wave and negative refraction. Carbonnel et al verified the backward wave propagation in [10]. Negative refraction was confirmed experimentally by Pendry and Smith in [11-12]. A number of new structures such as spiral multi-split, omega shape and S-shape have been proposed in [13] which show LHM characteristics. In [14-16], authors analyzed the SRR structures to conclude dependence of effective material parameters on geometrical dimensions of metamaterial structure to upgrade the properties of the microwave devices.

It is well known fact that antenna gives low efficiency when its size is reduced below Chu limit [17-20]. Because of this reason, antenna design has been a big challenge for researchers. Many miniaturization techniques are available in literature like insertion of slots on 
the radiating patch [21] and design of fractal based antenna [22] etc., but this problem can be solved through magnetic permeability enhanced metamaterials which are used to design small antennas below Chu limit with considerable size reduction [5], [23-24].

In this paper, a new left hand metamaterial structure consisting of double turn spiral resonator (DTSR) and two capacitively loaded strips(CLS) of copper, is modelled, optimized and simulated using Finite Element Method (FEM) based Ansoft HFSS software to prove the negative refraction property. The proposed structure has a single spiral resonator along with CLS which is different from work done in [25] where double cut SRRs are being used. The use of spiral resonator (SR) is proposed in this paper because the spiral resonators use the unit cell area effectively and have significant potential to reduce the electrical size of the metamaterial unit cell than the conventional square SRR structures [5, 23]. We preferred a spiral loop as it uses less area to provide equivalent capacitance while simultaneously provide additional inductance and hence additional permeability [16, 23].

This paper is organised in four sections. After discussion of previous work done in Introduction, Section 2 discusses proposed design of LHM structure. Section 3 presents simulation methodology of LHM unit cell inside a waveguide with suitable boundary conditions and excitations. Section 4 presents numerically analyzed results and discussions. Section 5 gives conclusion of paper.

\section{Proposed Design of LHM Structure}

The proposed LHM unit cell structure consisting a double turn spiral resonator (DTSR) and two capacitively loaded strips (CLS) on both sides (left and right side) of Spiral Resonator (SR) is shown in Figure 1. The geometrical parameters of SR and CLS are given in Table 1. The proposed structure is patterned on FR-4 substrate with permittivity 4.7, thickness $1.6 \mathrm{~mm}$, and loss tangent of 0.019 .

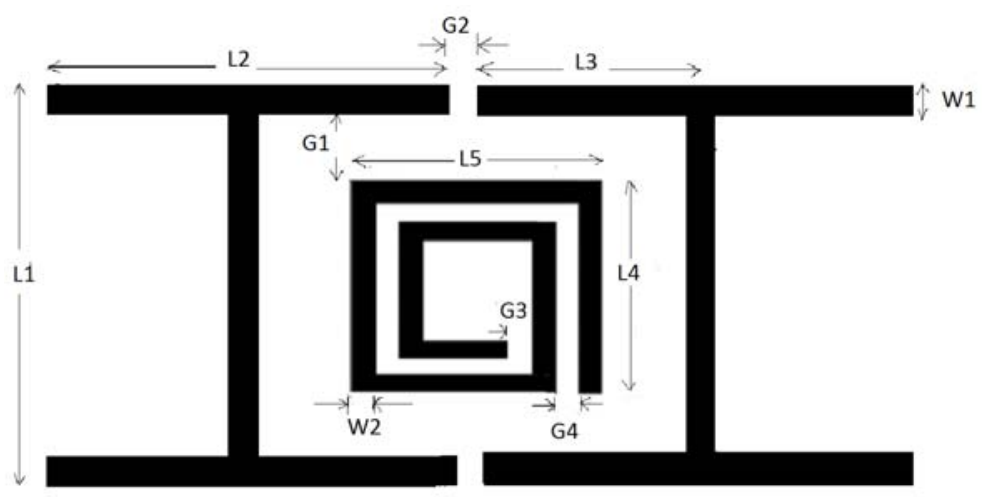

Figure 1. LHM unit cell structure geometry

Table 1. Geometrical Parameters of the Proposed LHM Unit Cell

\begin{tabular}{lll}
\hline S.N. & \multicolumn{1}{c}{ Dimension of LHM Structure } & Units (mm) \\
\hline 1 & Height of two capacitance strips (L1) & 17.52 \\
2 & Length of full CLS (L2) & 15.2 \\
3 & Length of half strip (L3) & 7.6 \\
4 & Gap between SR and CLS (G1) & 3.5 \\
5 & Gap between two CLS strips (G2) & 1.16 \\
6 & Width of CLS (W1) & 1.16 \\
7 & Width or metallic thickness of a turn of SR (W2) & 0.45 \\
8 & Gap or split of SR (G3) & 0.45 \\
9 & Spacing between two turns of SR (G4) & 0.45 \\
10 & Length of outer arm of SR (L5 and L4) & 8.19 \\
\hline
\end{tabular}

TELKOMNIKA Vol. 15, No. 3, September $2015: 497-503$ 
Metallic length of DTSR and CLS is equivalent to inductive coil. The gap in SR and CLS generates parallel plate capacitor. An electric circular current is induced in the metallic ring when placed in a time varying magnetic field. Thus, SR is a resonator which couples to a perpendicular magnetic field and can be characterized by the effective capacitance of the gap and effective inductance of the loop defined by the metallic ring. The CLS introduces an extra capacitance [25]. We can tune several parameters of SR like size of SR, spacing between the rings, size of the split [15], thickness of substrate, length, width and height of CLS and gap between two CLS to control magnetic and electric resonance.

\section{Simulation Methodology of LHM in Waveguide}

We put the proposed LHM unit cell in a waveguide as shown in Figure 2 and simulate the metamaterial structure. Perfect electric conductor (PEC) boundary conditions are assigned on the z-faces of the unit cell. The perfect magnetic conductor (PMC) boundary conditions are applied on the $y$-faces of the unit cell. The two wave ports 1 and 2 are assigned along each of the substrate line on the $x$-faces form $-x$ to $x$ direction.

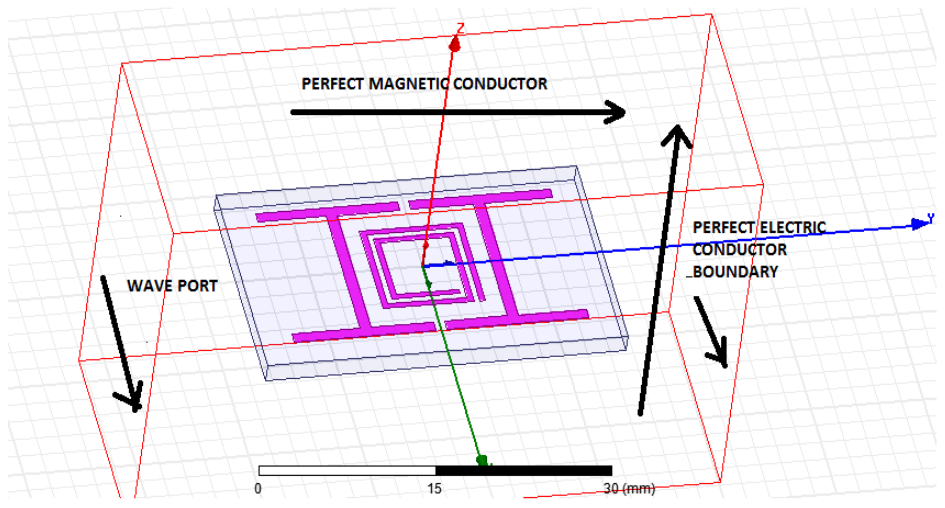

Figure 2. Boundaries and excitations in LHM unit cell

The metamaterial unit cell is modelled in HFSS and allocated suitable boundary conditions for far-field calculation to get consistent results. After modeling, the adaptive meshing is applied to problem domain. The adaptive meshing algorithm looks for the largest gradients in the $E$ field or error and then subdivides the mesh in these areas or regions. For the simulation, 25 passes have been taken with an error tolerance of $2 \%$. HFSS compares the S-Parameters from the current mesh to the results of the preceding mesh. With each adaptive pass, maximum $30 \%$ refinement per pass is achieved in solutions. Once the user defined error tolerance is attained, then the solution get converged and the current or preceding mesh is used to execute a frequency sweep.

The effective permeability $\left(\mu_{r}\right)$ and effective permittivity $\left(\varepsilon_{r}\right)$ of an equivalent metamaterial can be determined using Nicolson-Ross-Weir approach [20]. This method is applied to retrieve effective material parameters and MATLAB code is written to implement the following equations:

$$
\begin{aligned}
& V_{1}=S_{21}+S_{11} \\
& V_{2}=S_{21}-S_{11}
\end{aligned}
$$

The complex terms, $V_{1}$ and $V_{2}$ symbolize the summation and difference of Sparameters and are estimated using Equations (1) and (2) by exporting values of transmission coefficient $\left(S_{21}\right)$ and reflection coefficient $\left(S_{11}\right)$.

$$
k=\left(\frac{\omega}{c}\right) * \sqrt{\mu_{r} \varepsilon_{r}}=k_{0} \sqrt{\mu_{r} \varepsilon_{r}}
$$


Where $k$ is complex wave number, $\omega$ is angular frequency, $k_{0}$ is wave number in free space $\left(k_{0}=\omega / c\right), \mu_{r}$ is effective permeability, $\varepsilon_{r}$ is effective permittivity of equivalent metamaterial and $c$ is speed of light.

$$
\begin{aligned}
& \mu_{r}=\frac{2}{j k_{0} d} \frac{1-V_{2}}{1+V_{2}} \\
& \varepsilon_{r}=\frac{2}{j k_{0} d} \quad \frac{1-V_{1}}{1+V_{1}}
\end{aligned}
$$

$d$ is thickness of substrate.

By putting the Equations (1) and (2) in eqns. (4) and (5), we can get effective permeability $\left(\mu_{r}\right)$ and effective permittivity $\left(\varepsilon_{r}\right)$.

\section{Results and discussion}

A Full wave simulation of the proposed LHM unit cell in a waveguide is performed with electromagnetic solver. After applying appropriate boundary conditions and excitations, as mentioned in Section 3, the model is then executed to verify its metamaterial features. The transmission and reflection characteristics in terms of S-parameters are plotted for the proposed LHM structure for validating the performance.

\subsection{Reflection Coefficient $\left(S_{11}\right)$ and Transmission coefficient $\left(S_{21}\right)$}

Figure 3 shows reflection coefficient $\left(S_{11}\right)$ and transmission coefficient $\left(S_{21}\right)$ characteristics of LHM w.r.t. frequency. It can be noticed from the simulated results that there is strong reflection of $-23.7 \mathrm{~dB}$ at $1.84 \mathrm{GHz}$. This signifies that proposed LHM resonates at 1.84 $\mathrm{GHz}$. Resonance occurs at the frequency close to the frequency location where the logarithmic transmission has a minimum value. The first transmission minimum for the proposed structure is $-37.8 \mathrm{~dB}$ at $2.12 \mathrm{GHz}$.

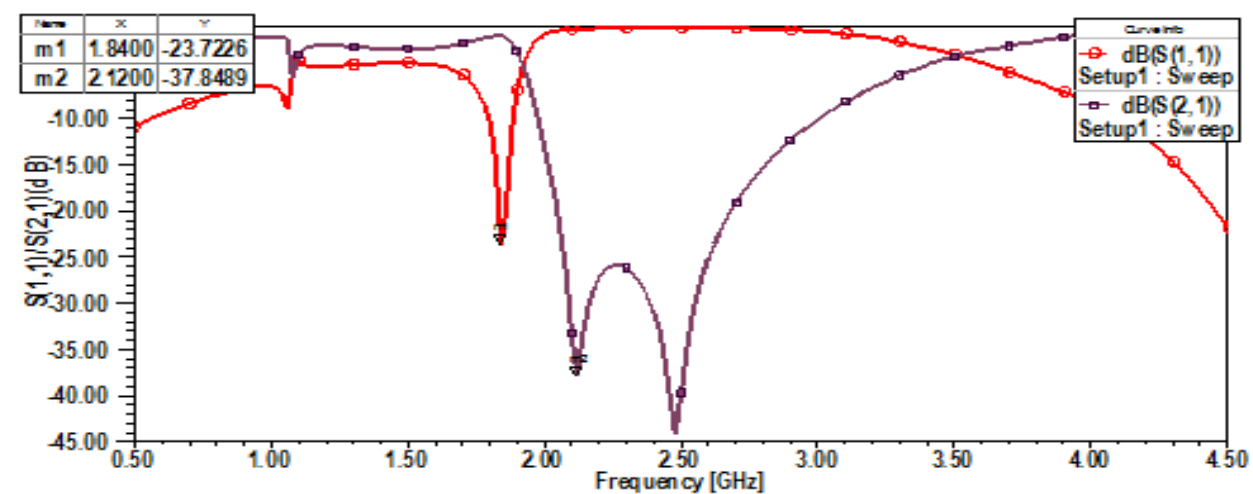

Figure 3. Transmission coefficient $\left(S_{21}\right)$ and reflection coefficient $\left(S_{11}\right)$

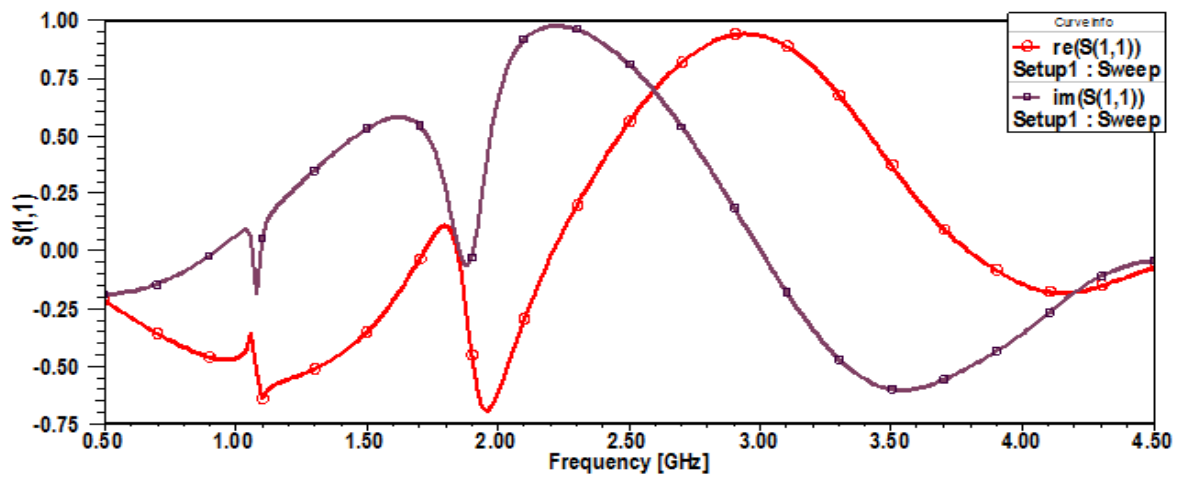

Figure 4. Real and imaginary part of reflection coefficient $\left(S_{11}\right)$

TELKOMNIKA Vol. 15, No. 3, September 2015 : $497-503$ 
The dip in the phase of $S_{21}$ is observed for the designed LHM structure and the negative refraction region is identified. Real and imaginary parts of $S_{11}$ are depicted in Figure 4 and Figure 5 respectively. These values are used to evaluate the negative characteristics of permeability and permittivity for the proposed LHM structure.

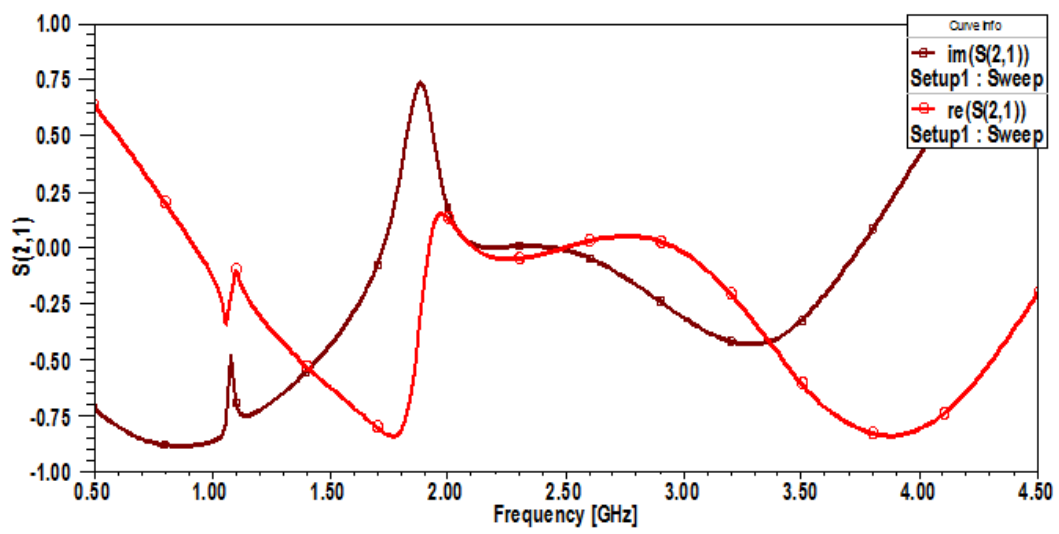

Figure 5. Real and imaginary part of transmission coefficient $\left(S_{21}\right)$

The magnitude and phase of $S_{11}$ and $S_{21}$ are shown in Figure 6 and Figure 7 respectively. The reversal of phase of $S_{11}$ and $S_{21}$ at particular frequency validates the metamaterial behaviour of SR.

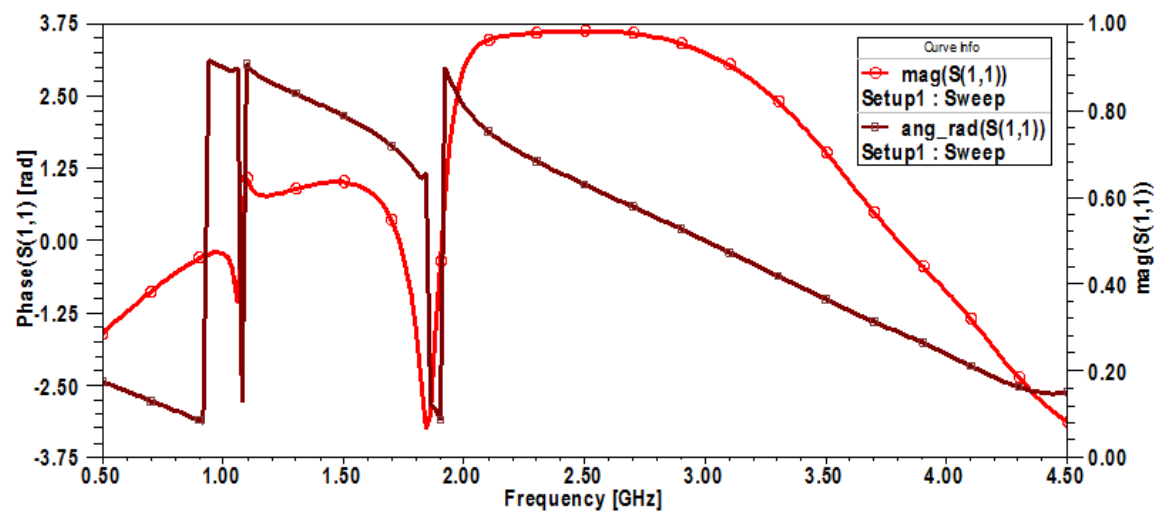

Figure 6. Magnitude and phase of reflection coefficient $\left(S_{11}\right)$

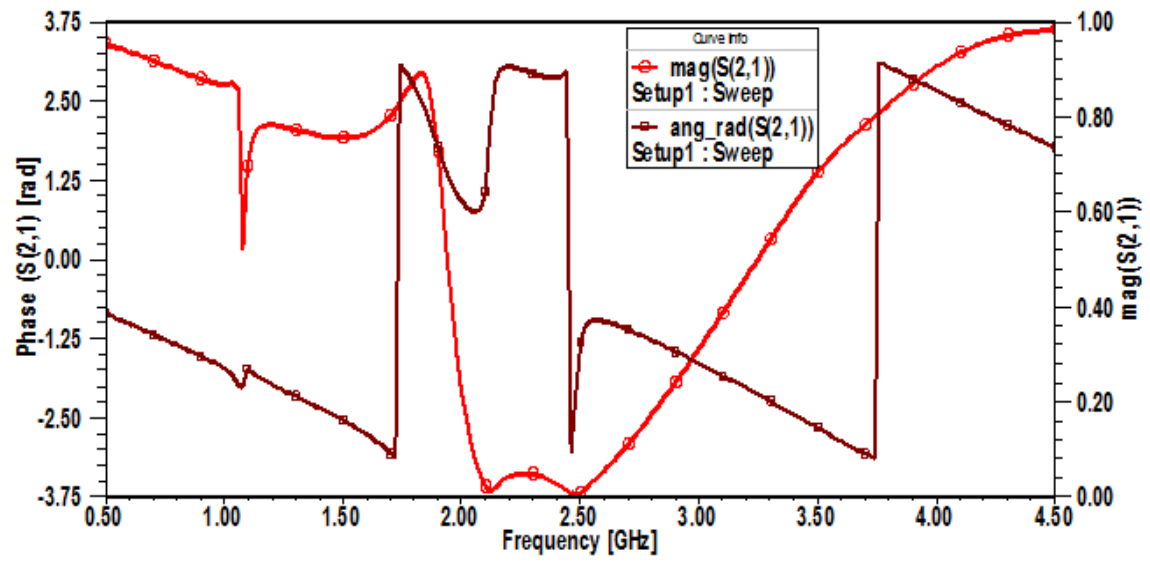

Figure 7. Magnitude and phase of transmission coefficient $\left(S_{21}\right)$ 


\subsection{Effective Permeability and Effective Permittivity}

Figure 8 depicts the extracted real part of permeability and permittivity. Red curve shows real part of permeability. Green line presents the real part of permittivity. To evaluate the effective permeability and effective permittivity, first MATLAB code is generated. Then by exporting the values of $S_{11}$ and $S_{21}$ obtained from Figure 4 and Figure 5 in MATLAB and implementation of Equations (4) and (5), we evaluate effective permeability, $\mu_{r}$ and effective permittivity, $\varepsilon_{r}$ to verify the metamaterial properties.

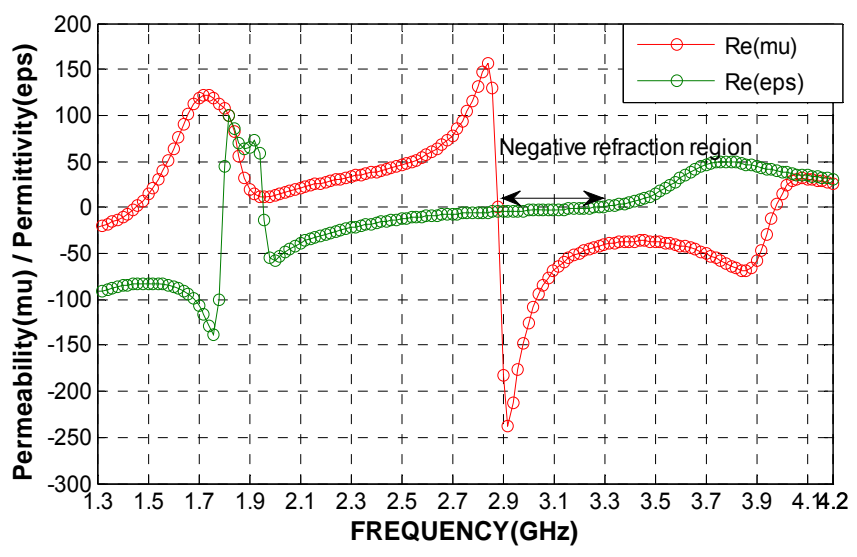

Figure 8. Permittivity, Permeability and Negative refraction region of LHM Unit cell

According to the theory of metamaterials, the real part of $\varepsilon_{r}$ and $\mu_{r}$ must be negative for the proposed LHM structure. It can be observed from the plot that the negative values of permittivity and permeability are achieved for the proposed structure. The real part of the permittivity is apparently negative from $1.95-3.28 \mathrm{GHz}$. These are the electric plasma frequencies for proposed LHM structure. The negative real part of the permeability lies between $2.88-3.98 \mathrm{GHz}$. These are magnetic plasma frequencies for the proposed LHM. The negative band of refractive index for the proposed LHM exists at overlapped region of magnetic plasma frequencies and electrical plasma frequency shown in Figure 8. For the proposed structure, the permeability and permittivity simultaneously become negative for a frequency range of 2.88 $\mathrm{GHz}$ to $3.28 \mathrm{GHz}$. Hence it is marked that the proposed LHM exhibits negative refraction in 2.88 $\mathrm{GHz}$ to $3.28 \mathrm{GHz}$ frequency range.

\section{Conclusion}

This work successfully demonstrates the metamaterial properties of proposed LHM unit cell. This new structure exhibits double negative properties from $2.88 \mathrm{GHz}$ to $3.28 \mathrm{GHz}$. Hence negative refraction is observed with this LHM in this frequency range. This work can be extended to get periodic structure of this unit cell and can be used as superstrate for microstrip antenna to enhance its performance characteristics. This structure can also be used to construct new functional devices such as electromagnetic filters, antennas and waveguides.

\section{References}

[1] Mookiah Prathaban, Dandekar Kapil R. Metamaterial Substrate Antenna Array for MIMO Communication System. IEEE Transaction on Antennas and Propagation. 2009; 57(10): 3283-3292.

[2] Sulaiman Ahmad A, Othman Adib, Jusoh Mohd H, Baba Noor H, Awang Robi'atun A Ain, Mohd FA. Small Patch Antenna on Omega Structure Metamaterial. European Journal of Scientific Research, 2010; 43(4): 527-537.

[3] Enoch S, Tayeb G, Sabouroux P, Guerin N, Vincent P. A metamaterial for directive emission. Phys. Rev. Lett. 2000; 89(21): 213-902.

[4] Lim S, Caloz C, Itoh T. Metamaterial-based electronically controlled transmission-line structure as a novel leaky-wave antenna with tunable radiation angle and beamwidth. IEEE Trans. Microw. Theory Tech. 2005; 53(1): 161-173. 
[5] Buell K, Mosallaei H, Sarabandi K. A substrate for small patch antennas providing tunable miniaturization factors. IEEE Trans. Microw. Theory Tech. 2006; 54(1): 135-146.

[6] Veselago VG. The electrodynamics of substances with simultaneously negative values of ' $\varepsilon$, and $\mu$ '. Soviet Physics- USPEKHI. 1968; 47: 509-514.

[7] Pendry JB, Holden AJ, Robbins DJ, Stewarts WJ. Low Frequency Plasmons for Thin-Wire Structure. J. Phys. Condens. Matter. 1998; 10: 4785-4809.

[8] Holden J, Robbins DJ, Stewart WJ. Magnetism from conductors and enhanced non-linear phenomena. IEEE Trans. Microwave Theory Technol. 1999; 47: 2075-2084.

[9] Shelby RA, Smith DR, Shultz S. Experimental verification of a negative index of refraction. Science. 2001; 292: 77-79.

[10] Carbonnel J, Rogla LJ, Boria VE, Lippens D. Design and Experimental verification of backward-wave propagation in periodic waveguide structures. IEEE Transactions on Microwave Theory and Techniques. 2006; 54(4): 1527-1533.

[11] Pendry JB, Smith DR. Reversing light: Negative Refraction. Physics Today. 2003: 1-8.

[12] Pendry JB. Negative refraction makes a perfect lens. Physics Review letters. 2000; 85(18): 39663969.

[13] Wu BI, Wang W, Pacheco J, Chen X, Grzegorczyk TM, Kong JA. A study of using metamaterials as antenna substrate to enhance gain. Progress in Electromagnetic Research. 2005; 51: 295-328.

[14] Rajni, Marwaha, Anupama. Analysis of magnetic resonance in metamaterial structure. Proceedings of COMSOL Conference 2011. Bangalore. 2011.

[15] Rajni, Marwaha A. Role of Geometry of Split Ring Resonators in Magnetic Resonance of Metamaterials. International Journal of Electronics and Communication Engineering \& Technology (IJECET). 2013; 4(7): 279-285.

[16] Bilotti Filiberto, Toscan Alessandro, Vegni Lucio. Design of Spiral and Multiple Split Ring Resonators for the realization of Miniaturized Metamaterial Samples. IEEE transactions on antennas and propagation. 2007; 55(8): 2258-2267.

[17] Chu LJ. Physical limitations on Omni-directional antennas. Journal of Applied Physics. 1948; 19: 1163-1175.

[18] Ziolkowski Richard W, Erentok A. At and below the Chu limit: passive and active Broad Bandwidth Metamaterial Based Electrically Small Antennas. IET Microwave and Antennas Propagation. 2007; 1(1): 116-128.

[19] Ziolkowski, Richard W, Erentok A. Metamaterial based efficient electrically small antennas. IEEE Trans. on Antennas Propag. 2007; 54(7): 2113-2130.

[20] Joshi JG, Pattnaik SS, Devi Swapna, lohokare M. Electrically Small Patch Antenna Loaded with Metamaterial. IETE Journal of Research. 2010; 56(6): 373-379.

[21] Parashar KK. Design and Analysis of I-Slotted Rectangular Microstrip Patch Antenna for Wireless Application. International Journal of Electrical and Computer Engineering. 2014; 4(1): 31-36.

[22] Lodhi SS, Singhal PK, Thakare VV. Design and Analysis of Tripple Band Koch Fractal Yagi Uda Antenna. International Journal of Electrical and Computer Engineering (IJECE). 2013; 3(4): 456-460.

[23] Rajni, Marwaha Anupma, Kaur Gursharan. Metamaterial Inspired Circular Patch Antenna using Complementary Split Ring Resonator and Complementary Spiral Resonator. International Journal of Applied Engineering Research. 2015; 10(9): 22717-22727.

[24] Rajni, Marwaha Anupma, Kaur Gursharan. Metamaterial Inspired Patch Antenna by adding single layer Complementry split ring resonators for ISM band operation. International Journal of Electrical and Computer Engineering. 2015.

[25] Majid HA, Rahim MK, Masri T. Microstrip antenna's gain enhancement using left-handed Metamaterial structure. Progress in Electromagnetics Research. 2009; 8: 235-247. 